\title{
NR4A3 and CCL20 Clusters Dominate the Dynamic Gene Network of Peripheral CD146+ Blood Cells in the Early Stage of Acute Myocardial Infarction in Human.
}

\section{Yanhui Wang \\ Shandong University of Science and Technology \\ Chenxin Li \\ Shandong University of Science and Technology \\ Jessica M Stephenson \\ University of Texas Health Science Center at Houston}

\section{Sean P Marrelli}

University of Texas health science center at houston

\section{Yanming Kou}

Shandong University of Science and Technology

\section{Dazhi Meng}

Beijing University of Technology

Ting Wu ( $\nabla$ ting.wu@uth.tmc.edu )

University of Texas Health Science Center at Houston https://orcid.org/0000-0001-8152-1112

\section{Research}

Keywords: acute myocardial infarction (AMI), CD146, Pearson network, clustering coefficient, differential connectivity genes (DCGs)

Posted Date: December 28th, 2020

DOl: https://doi.org/10.21203/rs.3.rs-133154/v1

License: (c) (1) This work is licensed under a Creative Commons Attribution 4.0 International License. Read Full License 
$1 \quad N R 4 A 3$ and $C C L 20$ clusters dominate the dynamic gene network of peripheral $\mathrm{CD}_{146}^{+}$

2 blood cells in the early stage of acute myocardial infarction in human.

3 Yan-hui Wang ${ }^{1,{ }^{*}}, \mathrm{PhD}$; Chen-xin $\mathrm{Li}^{1}$, MS; Jessica M Stephenson ${ }^{2}$, BS; Sean P Marrelli ${ }^{2}, \mathrm{PhD}$;

$4 \quad$ Yan-ming $\mathrm{Kou}^{1}$, MS; Da-zhi Meng ${ }^{3, *}, \mathrm{MS}$; Ting $\mathrm{Wu}^{2}$ *, $\mathrm{PhD}$;

5

$6{ }^{1}$ College of Mathematics and Systems Science, Shandong University of Science and

7 Technology, Qingdao, China

$8{ }^{2}$ Department of neurology, University of Texas health science center at Houston, Houston, Tx,

9 USA

$10{ }^{3}$ College of Applied Science, Beijing University of Technology, Beijing, China

11

$12 *$ Corresponding authors

13 Yanhui Wang, tel: +86-15192542635, yanhuiwang2014@163.com, 579 Qianwangang Road,

14 Huangdao district, Qingdao, Shandong, 266590, China.

15 Dazhi Meng, tel: +86-13701377108,dzhmeng07@163.com, 100 Pingleyuan chaoyang district,

16 Beijing, 10024, China.

17 Ting Wu, tel: +1 5022998114, ting.wu@uth.tmc.edu, 6431 Fannin street, Houston, 77031,

18 USA.

19

20 Short title: CCL20 and NR4A3 lead CD146-mediated AMI pathology. 


\section{Abstract}

23 Background: CD146 is a tight junction associated molecule involved in maintaining

24 endothelial barrier and balancing immune-inflammation response in cardiovascular disease.

25 Notably, the peripheral $\mathrm{CD} 146^{+}$cells significantly upsurge under vessel dyshomeostasis like acute myocardial injury (AMI), appearing to be promising therapeutic targets. In this study, in a new view of gene correlation, we aim at deciphering the underlying complex mechanism of $\mathrm{CD}_{146^{+}}$cells in the development of AMI.

Methods: Transcription dataset GSE 66360 of CD146 ${ }^{+}$blood cells from clinical subjects were downloaded from NCBI. Pearson networks were constructed and the clustering coefficients were calculated to disclose the differential connectivity genes (DCGs). Analysis of gene connectivity and gene expression was performed to reveal the hub genes and hub genes clusters followed by gene enrichment analysis.

34 Results and conclusions: Among the total 23520 genes, 27 genes out of 126 differential expression genes are identified as DCGs. Those DCGs normally stay in the peripheral of networks while transfer to the functional central position under AMI situation. Moreover, it is revealed that DCGs spontaneously crowd together into two functional models, CCL20 cluster and NR4A3 cluster, influencing the CD146-mediated signaling pathways during the pathology of AMI for the first time.

41 Keywords: acute myocardial infarction (AMI), CD146, Pearson network, clustering coefficient, differential connectivity genes (DCGs) 


\section{Introduction}

44 Cluster of differentiation 146 (CD146) / melanoma cell associated molecule is an essential

45 immunoglobulin-like protein initially discovered in metastatic melanoma (1). It locates at

46 endothelial tight junctions across all vessel beds, mediating physiological and pathological

47 events under vascular dyshomeostasis $(2,3)$. Pioneering researchers regard CD146 as a

48 historical marker for isolating circulation endothelial cells that sloughed off the inflamed

49 vasculature (4). Over several decades, CD146 has also been discovered in other cell types

50 including mesenchymal stem cells (5), endothelial progenitor cells (6), macrophages (7), $\mathrm{T}$

51 helper 17 cells (8), B lymphocytes (9), T lymphocytes $(9,10)$, and natural killer cells (9). The

$52 \mathrm{CD} 146^{+}$circulating cells occupy about $2 \%$ of peripheral mononuclear cells in healthy

53 individuals (9) and most notably, this percentage increases in certain conditions associated with

54 vascular dysfunction for instance myocardial infarction, connective tissue diseases, and cancers

55 (6, 11-13). Moreover, CD146 activated T cells have shown an enhanced ability to interact with

56 endothelium in adhesion, rolling, and transmigration, evidenced by human and murine studies

$57(14,15)$. Given its multi-function in vessel structure, angiogenesis, and lymphocyte activation

58 and its enabled detection in the bloodstream, CD146 appears to be a potential target for vascular

59 disorders (16-18).

60 Complex networks are of great interest to researchers in the fields of computational biology

61 and bioinformatics (19-21). It has been gradually extended from initial gene comparison to

62 protein-protein network modeling, to protein-genetic investigation, and up to the disease- 
63 disease association exploration (22). Most of the successful bioinformatics approaches that

64 identify the initial key genes, however, have based on sole gene expression comparison and accordingly the top differential expression genes (DEGs) forward to mechanism validation without paying attention to the gene interaction rearrangement $(23,24)$. Instead, the hub-

67 structured network is an important motif that is, to our best knowledge, leading the genomewide association characterization in complex networks $(25,26)$. It generates the structure view angle to present the innermost gene-gene interaction, thus giving a comprehensive understanding of underlying mechanisms of disorders.

Acute myocardial injury (AMI) dataset GSE 66360 rests on the performance of CD146 populations during the AMI early development (12, 27-29). In this paper, we try to decipher gene reassemble with the correlation network structure parameter analysis $(30,31)$, and extract optimal genes collection, the differential connectivity genes (DCGs), and reveal functional gene clusters which likely leading the pathogenesis of peripheral CD146 ${ }^{+}$blood cells during the AMI development in human for the first time.

\section{Materials and methods}

\subsection{Data}

The GSE66360 (12) gene transcription profile data of human AMI in the NCBI database was selected as the primary interest. Clinical subjects including fifty healthy individuals and forty-

81 nine AMI patient subjects were recruited in the original investment by the Topol group. To 
gather the data, $\mathrm{CD} 146^{+}$cells were obtained by CD146-based magnetic immunoisolation from

83 the subjects' blood samples. RNA samples were isolated from the CD146 ${ }^{+}$cells and processed

84 microarray by Affymetrix human U133 Plus 2.0 array. In this study, two cohorts were formed,

85 a discovery cohort, consisting of twenty-two healthy subjects (control group) and twenty-one

86 AMI patients (AMI group), which were used for the discovery of genes and appropriate testing

87 methods, along with a validation cohort, consisting of twenty-eight healthy subjects and twenty-eight AMI patients, which were used for the validation of the genes and methods discovered in the other cohort. No data was excluded from the original databases used during 90 this study.

\subsection{Study design}

92 Firstly, using the hypothesis test, we distinguished DEGs based on the gene expression profile

93 in the discovery cohort and then verified in the validation cohort. Secondly, the gene networks

94 of DEGs were constructed based on the Pearson coefficients, followed by the network separation assessment. Thirdly, the clustering coefficient, which is a parameter indicating gene connectivity, was calculated for each DEG under each gene network (32). Accordingly, genes

97 with a clustering coefficient that represent a consistent increase in the AMI group among different cohorts were labeled as DCGs. Finally, two-dimensional analysis of gene connectivity and expression was employed, for identifying the hub gene clusters, alongside performing the gene enrichment analysis (Figure 1).

\subsection{Identify DEGs}


102 A total of 23520 genes were screened in each sample. The hypothesis test was used to screen

103 the DEGs between control and AMI group (33). The method primarily gave weight for the

104 distribution shape of the expression spectrum. If the distribution shape was different between

105 the two groups, then the gene expression was different and the significance level was $\alpha_{1}$. If not,

106 a normal distribution test (significance level $\alpha_{2}$ ) and homogeneity test of variance (significance

107 level $\alpha_{3}$ ) would be carried out. t-test or welch's t-test (significance level $\alpha_{4}$ ) was used for normal

108 distribution; Rank sum test (significance level $\alpha_{4}$ ) was used for abnormal distribution with a

109 similar distribution of expression spectrum (33). We defined $\alpha_{1}=0.00001, \alpha_{2}=0.00002, \alpha_{3}=$

1100.00001 , and $\alpha_{4}=0.00001$ as the significant level of the hypothesis test.

1112.4 Clustering coefficient

112 A local clustering coefficient was introduced to measure the compactness, or the connectivity

113 of genes within a suspected cluster, of a complete array formed by the adjacent nodes within a

114 network (34). To clarify, assume that a node $\mathrm{i}$ in a network was connected to $\mathrm{k}_{\mathrm{i}}$ nodes. The $\mathrm{k}_{\mathrm{i}}$

115 nodes were called neighbors of node $i$. The ratio of the actual number $E_{i}$ of edges and the total

116 number $\mathrm{k}_{\mathrm{i}}\left(\mathrm{k}_{\mathrm{i}}-1\right) / 2$ of possible edges between $\mathrm{k}_{\mathrm{i}}$ nodes were defined as the clustering coefficient,

$117 \mathrm{C}_{\mathrm{i}}$, of node $\mathrm{i}$, that is, $\mathrm{C}_{\mathrm{i}}=2 \mathrm{E}_{\mathrm{i}} /\left(\mathrm{k}_{\mathrm{i}}\left(\mathrm{k}_{\mathrm{i}}-1\right)\right)$.

$118 \quad 2.5$ Pearson network construction and assessment

119 Pearson correlation networks of DEGs were constructed according to the absolute value of

120 Pearson coefficients. Two genes were considered correlated if the absolute value of the Pearson 
121 coefficient was greater than the threshold $\mathrm{x}(0<\mathrm{x}<1)$, then a line could be drawn between the

122 two genes. In the cases when genes were not correlated, there would be no link in the network

123 and thus no line could be drawn. Gene clusters were determined by examining the clustering

124 coefficients, and those with a non-zero value could be labeled clusters. Gene clusters represent

125 a functional module as a whole with varying degrees of connectivity; while a degree describes

126 the number of genes connected to one another. The average clustering coefficients of DEGs

127 were calculated to evaluate the overall separation of the control and AMI networks. The method

128 was implemented in $\mathrm{R}$ i386 3.6.2.

129 Natural biological networks are scale-free networks and the degree distributions follow the

130 power-law exponential distribution index range $2 \sim 3(35,36)$. We indicated the gene networks

131 under threshold 0.5 and 0.7 since the power-law indexes of degree distribution in discovery

132 cohort were in the range of $2 \sim 3$ and presented the corresponding networks in validation cohort

133 in this study (Supplement Table 1).

1342.6 Identify DCGs

135 In the analysis of network connection parameters, the greater the difference between the control

136 and AMI group, the higher the correlation with AMI. The following is describing our unique

137 identifying method. Assume that the average clustering coefficient of the control group and

138 AMI group could be separated at the threshold [0.1, 0.9]. First, the clustering coefficient of

139 each gene in the Pearson correlation networks of the control group and AMI group, under the

140 threshold $0.1 \sim 0.9$, were calculated with step length 0.1 . Secondly, the average clustering 
141 coefficient of each gene cross threshold $0.4 \sim 0.8$ was calculated to compare changes in

142 connectivity between the two groups within the validation and discovery cohorts. Finally, if

143 clustering coefficient differences in discovery cohort and validation cohorts were consistently

144 greater than 0.1 between the AMI group and the control group, the genes were identified as

145 candidates for DCGs.

146 To test the reliability of the proposed candidate genes across different datasets, we expanded

147 our method to a combination cohort, which included all subjects in both discovery and

148 validation cohorts. Increasing the number of subjects, but also introducing some variation in

149 the data due to the less categorized subject population. The overall network between the control

150 and the AMI groups were still separable through threshold $0.4 \sim 0.8$ (data not shown). While

151 having 27 out of 39 candidate genes still showing clustering coefficient differences were

152 greater than 0.1 in this combination cohort were define as DCGs.

$153 \quad 2.7$ Gene set enrichment

154 Gene set enrichment was performed by the STRING server. Biological process, Reactome

155 pathways and protein-protein association networks were generated for CCL20 cluster, NR4A3

156 cluster and DCGs.

$157 \quad 2.8$ Graphs

158 Heatmaps of DEGs and DCGs were generated by using heatmap.2 function in the gplots

159 package. Networks were computed by using igraph::graph.data.frame function. Layout 
160 algorithm of layout.kamada.kawai was used for visualizing the overall DEGs networks and the

161 connections for individual genes. Layout algorithm of layout.circle was used to visualize the

162 gene connections within DEGs and DCGs in circle view. Cytoscape network function was used

163 to generate the clustered DCGs networks.

\section{3. Results}

\section{$165 \quad 3.1$ DEGs identification}

166 In our initial analysis, 126 out of 23520 genes are significantly altered in the AMI group

167 compare to the control group in discovery cohort, defined as DEGs, with the majority (79 of

168 126) demonstrate an up-regulation feature (Figure 2A). And those genes show a similar

169 expression pattern in the validation cohort (Figure 2B).

$170 \quad 3.2$ Assessment of DEGs' networks

171 The overall gene networks of DEGs in the control group and the AMI group are distinctly

172 independent through a large range of thresholds in discovery cohort and validation cohort

173 (Figure 3A). The networks in discovery cohort are separable through threshold $0.1 \sim 0.9$ and

174 in validation cohort are separable through $0.1 \sim 0.8$. The average separable widths of the

175 discovery cohort and validation cohort are 0.218 and 0.0518 , respectively. The validation

176 cohort shows a narrower split range possibly attributed to the variations between two cohorts,

177 for instance, the differential sample size, age, and co-morbid disorders. 
In addition, the gene connections within DEGs' networks in the AMI group are more complex

179 than those in the control group in both cohorts (Figure 3C). In discovery cohort, the number

180 of gene clusters within AMI network gradually decreases from 125 to 67, when threshold

181 increases from 0.4 to 0.8 , while it more sharply decreases from 123 to 17 in the control group

182 (Figure 3B). Similarly, the clusters decline with a lower slope in the AMI group compared to

183 the control group in validation cohort (Figure 3B).

184 The data described above suggest that gene networks of DEGs are largely and consistently

185 disturbed by AMI stimulation as is seen in two independent cohorts, verifying our findings.

186 Thus, DEGs and DEGs-networks are mathematically reliable and hereafter can be set as the

187 foundation for in-depth gene interaction data mining.

$188 \quad 3.3$ DCGs identification and connectivity analysis

189 Beyond DEGs, we identified 27 genes as DCGs whose clustering coefficients difference is

190 greater than 0.1 in the discovery cohort, the validation cohort, and the extended combination

191 cohort (Figure 4A, Supplement Table 2). The sub-networks of DCGs present obvious tighter

192 connections in the AMI group compare to the control group, in both discovery and validation

193 cohorts (Figure 4C). When threshold increased from 0.4 to 0.8 , the average degree of DCGs

194 progressively decreases from 16.0 to 4.30 in the AMI group, while it decreases from 7.93 to 0

195 in the control group in discovery cohort (Figure 4D). Similarly, in validation cohort, this

196 number decreases from 19.9 to 1.41 in the AMI group while from 3.26 to 0 in the control group 
197 (Figure 4D). Besides clustering coefficients, gene expression of those DCGs is showing a

198 steady increase in the AMI group in discovery and validation cohorts (Figure 4B).

199 Therefore, we propose that the networks' differential of all DEGs largely attributes to the 200 connection changes within DCGs. As visualized in kamada-kawai layout, the DCGs randomly

201 participate in the DEGs' network and connect to a few genes under a normal steady state.

202 However, they appear to interact with more functional genes and shift into central positions

203 after AMI in both discovery and validation cohorts (Figure 5).

2043.4 Two-dimensional analysis of gene connectivity and gene expression

205 Since the power-law indexes of degree distribution in the discovery are in the range of natural

206 network, we regard the discovery cohort as a more precise dataset and it is selected for the

207 following analysis. The average clustering coefficient and gene expression of DCGs are plotted

208 into a scatterplot for the two-dimensional analysis (Figure 6A). NR4A3 and CCL20 present

209 high levels of clustering coefficient and gene expression changes, defined as $\mathrm{CC}^{\text {high }} \mathrm{GeExp}{ }^{\text {high }}$

210 genes. SOCS3, FOSL2, PLIN2 are the genes that were found to have high clustering coefficient

211 changes (fold change $>2$ ) with low expression change, defined as $\mathrm{CC}^{\text {high }} \mathrm{GeExp}^{\text {low }}$ genes; while

212 IL1R2, NLRP3, ANXA3 and AC079305.10 are the genes that were found to have high

213 expression changes (fold change $>0.4$ ) with low clustering coefficient changes, defined as

$214 \mathrm{CC}^{\text {low }} \mathrm{GeExp}^{\text {high }}$ genes. All information on these genes is shown in Table 1. Subgraphs of

$215 N R 4 A 3, C C L 20$, and other DCGs provide pieces of evidence that support their increased gene

216 connectivity after AMI in the discovery cohort (Figure 6C, Supplement Figure 1A and 1B). 
218 Zooming into the subgraphs of individual genes, we reveal that DCGs stay "non-activated" in

219 the control group (Figure 7A). Interestingly, they appear to be spontaneously gathering 220 together as two separate clusters after AMI stimulation (Figure 7B, Supplement Figure 1A

221 and 1B). CCL20 connects with SKIL, MMP9, ITPRIP, ANXA3, GLUL, CXCL16, IL1R2,

222 TMCC3, NLRP3, PYGL, RNF144B, BCL6, LILRB2, CLEC4E, FCER1G, and AC079305.10,

223 identify as the CCL20 cluster. NR4A3 connects with NR4A2, FOSL2, CDKN1A, SOCS3,

224 GABARAPL1, ITPRIP, SYTL3, PELI1, MAP3K8, and PLIN2, identify as the NR4A3 cluster.

225 While there are overlapping genes between clusters, ITPRIP, SKIL and MAPK38 are the

226 intermediate genes that connect both clusters according to their subgraphs (Supplement

227 Figure 1B). The $C^{\text {high }} \mathrm{GeExp}^{\text {high }}$ gene, $N R 4 A 3$ or CCL20, serve as leading-like hub gene in

228 each cluster. The clustering coefficient fold changes of NR4A3 and CCL2O are 15.7 and 10.2,

229 respectively; and the gene expression fold changes are 0.379 and 0.422 , respectively.

\section{$230 \quad 3.6$ Gene enrichment}

231 Biological process analysis shows that DCGs are involving in response to organic substrates,

232 positive regulation of leukocyte activation, immune response, immune system process,

233 response to cytokine and regulation of cytokine production. The CCL20 cluster is essential to

234 the immune response, immune system process, and regulation of localization while the NR4A3

235 cluster is essential to cellular response to corticotropin-releasing hormone stimulus, positive

236 regulation of leukocyte activation, and regulation of apoptotic process (Table 2). 
237 Reactome pathway analysis revealed that DCGs are related to the immune system with regards

238 to tasks such as signaling by interleukins, namely interleukin-1, interleukin-4 and interleukin-

23913 signaling, the innate immune system and the dectin- 2 family. The CCL20 cluster is essential

240 to immune system, innate immune system, the dectin-2 family, and neutrophil degranulation

241 while the NR4A3 cluster is essential to RNA Polymerase II Transcription, Generic

242 Transcription Pathway, and MyD88 cascade initiated on plasma membrane (Table 2).

\section{4. Discussion}

244 CD146 is a junction-associated adhesion molecule that participates in immune and

245 inflammatory pathological processes in the initiation and development of vascular diseases (2).

246 CD146 activated leukocytes are recruited to the inflamed endothelial to induce the expression

247 of chemokines and cytokines and, in doing so, progressively destroys the blood vessel barrier.

248 Our study found that following AMI stimulation, in $\mathrm{CD}_{146^{+}}$human blood cells, 126 out of 249 total 23,520 genes show significant differential expression $(\mathrm{P}<0.0001)$ and among those, 27

250 genes show consistent connectivity changes and serve as DCGs. Unlike DEGs, DCGs are able

251 to not only aggregate gene expression but also encompass gene connectivity properties,

252 internally coupling into functional gene clusters - NR4A3 cluster and CCL2O cluster,

253 orchestrating the gene networks' entire dynamics in CD146 associated AMI pathophysiology

254 development. Meanwhile, NR4A3 and CCL20 are revealed as hub genes since they experienced

255 both connectivity and expression experienced significant changes after AMI stimuli.

256 Furthermore, gene enrichment analysis shows that the DCGs are involved in inflammation- 
257 immune response, with CCL20 being principal to the immune response and regulation of

258 localization; while, the $N R 4 A 3$ cluster is principal to leukocyte activation, apoptotic process,

259 and cellular response to corticotropin-releasing hormone stimulus; such findings align with the

260 well-known hypothesis that CD146 mediated inflammation plays an important role in the

261 pathogenesis of AMI.

262 The network structural parameter analysis method is applied to weave the gene-gene

263 correlation network. We identify DCGs which present steadily elevated connectivity under

264 AMI conditions, in both the discovery and validation cohorts, further confirming the

265 upregulation seen in the combination cohort (Supplement Table 2). As expected, the gene

266 expression of DCGs was increased after AMI, but was not distinguishable from DEGs solely

267 by expression signature (data not shown). NR4A3 and CCL20 as highlight hub genes were also

268 defined as AMI biomarkers after pre-filtering the co-morbidity relevant genes by the original

269 Topol group (12). SOCS3 tends to be the only "shared" AMI biomarker candidate revealed by

270 other groups in which the same GSE66360 dataset is included as one of their study objects (27,

271 28). Recognizing the CCL20, NR4A3, and SOCS3 as top DCGs substantiate previous outputs

272 and in turn, the validity of our method is enhanced. Therefore, we recommend the gene

273 connectivity analysis, along with gene expression signature, to be used as a powerful and

274 unbiased way for researchers to rank the importance of candidate DEGs.

$275 N R 4 A 3$ belongs to the $N R 4 A$ orphan nuclear receptor family (with $N R 4 A 2$ and $N R 4 A 1$ ), playing

276 a protective role in AMI development. The JM Penninger group reports that $N R 4 A 3$ is the 
277 highest-ranking gene in circulating human endothelial cells under atherosclerosis (37).

278 Transcription analysis of human left ventricular myocardium shows that $N R 4 A 3$ up-regulated

279 during ischemia and reperfusion in normal and chronic ischemic myocardium (38). Similarly,

$280 N R 4 A 3$ is found to be elevated 10-days post left anterior descending artery ligation ischemia

281 surgery in mice (39). Overexpression of $N R 4 A 3$ significantly reduces infarct size, preventing

282 deterioration of left ventricular function and repression of neutrophil infiltration in the heart of

283 mice after coronary artery ligation, relate to the activation of JAK2/STAT3 and the inhibition

284 of STAT3 dependent $N F-\kappa B$ signaling pathways (40). Additionally, it has to point out that the

$285 N R 4 A$ subgroup including $N R 4 A 3$ is an immediate early response gene induced by diverse

286 physiological, i.e., mechanical agitation, calcium, and inflammation cytokines (41). This

287 reinforces our data that, in the very early-stage AMI, NR4A3 has a significant 16-fold clustering

288 coefficient climb and $42 \%$ gene expression increase. Yet the nuclear factor $N R 4 A 3$ implications

289 in $\mathrm{CD}_{146^{+}}$related myocardial disorders remain a mystery.

290 CCL20, a C-C motif chemokine, is a chemoattractant for recruiting leukocytes to sites of injury

291 and inflammation (Figure 6B). CCL20 secretion is induced by pro-inflammatory chemokines

292 and cytokines, such as CXCL12, IL17, IL1 $\beta, I L 6$, and is in part related to JAK/STAT pathway

293 signaling in multiple cells (42-44). IL6 and soluble IL6 receptor stimulate STAT3 binding to

294 the CCL20 promotor and IL17 stimulate the phosphorylated $N F-\kappa B$ binding to the CCL2O

295 promoter in murine astrocytes, facilitate the neuroinflammation within central nervous system

296 (42). In addition, the co-expression of CCL20 receptor CCR6 and CD146 is a marker of effector 
297 memory Th17 cells, which mediate migration and is thought to be essential for inflammation

298 in human psoriasis (8). Moreover, it is reported that CCL20 level elevated in clinical patients'

299 serum with ischemic myocardial infarction $(45,46)$. In vitro study shows that CCL20

300 expression increase in $\mathrm{CD} 146^{+}$human mesenchymal stromal cells at the early pro-

301 inflammatory phase in fracture healing (47). Thus, we hypothesis that CCL20 binding its

302 receptor CCR6 is what drives the CD146-mediated vessel inflammation progress in early AMI

303 phase.

304 In terms of functional models, DCGs are self-organized into two clusters, the NR4A3 and

305 CCL20 clusters, with 18 genes and 12 genes in each cluster, respectively. All genes directly

306 link to its hub gene and partly link to adjacent genes as shown in Figure 7. Protein-protein

307 connection analyzed by STRING database produced a structure that is greatly similar to our

308 network pattern in which CCL20 connects with CXCL16, IL1R2, MMP9, NLRP3, BCL6

309 LILRB2, PELI1, CLEC4E, FCER1G, and NR4A3 connect with NR4A2, FOSL2, RNF144B,

$310 C D K N 1 A, \operatorname{SOCS3}$ (Supplement Figure 2). A few of gene-gene correlations within clusters are

311 stated in inflammatory diseases. MMP9 activation correlates with CCL20 expression in

312 astrocytes via Notch-1/Akt/NF- $\kappa B$ pathway promoting leukocyte migration cross blood-brain

313 barrier (48). NR4A2 and NR4A3 as orphan nuclear receptors mediate neutrophil number and

314 survival in chronic inflammatory signals multiple hematologic disorders (49-51). FOSL2 acts

315 as an activating protein-1 transcription factor promoting hematopoietic progenitor cell to 
316 macrophage and neutrophils in a SOCS3 dependent manner is reported (52). Nevertheless, most

317 of the cluster functions are rarely reported in AMI pathogenesis.

318 Taken together, we reveal that $N R 4 A 3$ and CCL20 clusters are novel functional modules in

$319 \mathrm{CD} 146^{+}$cells-mediated immuno-inflammatory balance, triggering increased susceptibility to 320 vascular deterioration and accelerating myocardial injury. $N R 4 A 3$ and CCL20 as hub genes

321 largely impact the early AMI development and can be promising targets for clinical diagnosis.

322 In-depth studies are necessary for understanding the mechanisms of peripheral CD146 ${ }^{+}$cells

323 in cardiovascular disease.

\section{Abbreviations list}

325 CD146: cluster of differentiation 146; DEGs: differential expression genes; DCGs: differential

326 connectivity genes; AMI: Acute myocardial injury; CC: clustering coefficient; GeExp: gene

327 expression.

328 Declaration

329 Ethics approval and consent to participate

330 Not required. The data has been available in the NCBI database.

331 Availability of data and materials

332 All data are included in the manuscript. 
333 Consent for publication

334 Not required.

335 Authors' contributions

336 Yanhui, Dazhi Meng and Ting Wu did the overall design of the study. Jessica M Stephenson

337 did the language editor for the manuscript. Chenxin Li contributed to the igraph figures. All

338 authors involved in the analysis of the data. All authors read and approved the final

339 manuscript.

340 Competing interests

341 No competing interests.

$342 \quad$ Funding

343 This research was supported by the Cultivation Project of Young and Innovative Talents in

344 Universities of Shandong Province and by NSFC (Grant No: 11501331).

345 Acknowledgments

346 The authors would like to acknowledge authors of primary study, the Topol group.

347

348 References 
350 Discrimination between benign and malignant cells of melanocytic lineage by two novel

351 antigens, a glycoprotein with a molecular weight of 113,000 and a protein with a molecular

352 weight of 76,000. Cancer research. 1987;47(3):841-5.

353 2. Shih IM. The role of CD146 (Mel - CAM) in biology and pathology. The Journal of

354 pathology. 1999;189(1):4-11.

355 3. Tu T, Zhang C, Yan H, Luo Y, Kong R, Wen P, et al. CD146 acts as a novel receptor for

356 netrin-1 in promoting angiogenesis and vascular development. Cell research. 2015;25(3):275-

35787.

358 4. Widemann A, Sabatier F, Arnaud L, Bonello L, AL - MASSARANI G, Paganelli F, et

359 al. CD146 - based immunomagnetic enrichment followed by multiparameter flow cytometry:

360 a new approach to counting circulating endothelial cells. Journal of thrombosis and

361 haemostasis. 2008;6(5):869-76.

362 5. Espagnolle N, Guilloton F, Deschaseaux F, Gadelorge M, Sensébé L, Bourin P. CD 146

363 expression on mesenchymal stem cells is associated with their vascular smooth muscle

364 commitment. Journal of cellular and molecular medicine. 2014;18(1):104-14.

365 6. Delorme B, Basire A, Gentile C, Sabatier F, Monsonis F, Desouches C, et al. Presence of 366 endothelial progenitor cells, distinct from mature endothelial cells, within human $\mathrm{CD}_{146^{\wedge}+}$

367 blood cells. THROMBOSIS AND HAEMOSTASIS-STUTTGART-. 2005;94(6):1270.

368 7. Luo Y, Duan H, Qian Y, Feng L, Wu Z, Wang F, et al. Macrophagic CD146 promotes

369 foam cell formation and retention during atherosclerosis. Cell research. 2017;27(3):352-72. 

and CD146 (MCAM) is a marker of effector memory T - helper 17 cells. The Journal of

372 dermatology. 2012;39(10):838-42.

373 9. Elshal MF, Khan SS, Takahashi Y, Solomon MA, McCoy JP. CD146 (Mel-CAM), an

374 adhesion marker of endothelial cells, is a novel marker of lymphocyte subset activation in 375 normal peripheral blood. Blood. 2005;106(8):2923-4.

376 10. Duda DG, Cohen KS, di Tomaso E, Au P, Klein RJ, Scadden DT, et al. Differential

377 CD146 expression on circulating versus tissue endothelial cells in rectal cancer patients:

378 implications for circulating endothelial and progenitor cells as biomarkers for antiangiogenic

379 therapy. Journal of clinical oncology: official journal of the American Society of Clinical

380 Oncology. 2006;24(9):1449.

381 11. Hadjinicolaou A, Wu L, Fang B, Watson P, Hall F, Busch R. Relationship of CD 146

382 expression to activation of circulating T cells: exploratory studies in healthy donors and

383 patients with connective tissue diseases. Clinical \& Experimental Immunology.

$384 \quad 2013 ; 174(1): 73-88$.

385 12. Muse ED, Kramer ER, Wang H, Barrett P, Parviz F, Novotny MA, et al. A whole blood 386 molecular signature for acute myocardial infarction. Scientific reports. 2017;7(1):1-9.

387 13. Fürstenberger G, Von Moos R, Senn H, Boneberg E. Real-time PCR of CD146 mRNA 388 in peripheral blood enables the relative quantification of circulating endothelial cells and is an 389 indicator of angiogenesis. British journal of cancer. 2005;93(7):793-8. 
391 melanoma cell adhesion molecule (MCAM)/CD146 in lymphocyte endothelium interaction:

MCAM/CD146 promotes rolling via microvilli induction in lymphocyte and is an endothelial

393 adhesion receptor. The Journal of Immunology. 2007;179(10):6673-85.

394 15. Dagur PK, McCoy Jr JP. Endothelial-binding, proinflammatory T cells identified by MCAM (CD146) expression: Characterization and role in human autoimmune diseases.

Autoimmunity reviews. 2015;14(5):415-22.

16. Leroyer AS, Blin MG, Bachelier R, Bardin N, Blot-Chabaud M, Dignat-George F.

CD146 (Cluster of Differentiation 146) An Adhesion Molecule Involved in Vessel

399 Homeostasis. Arteriosclerosis, thrombosis, and vascular biology. 2019;39(6):1026-33.

400 17. Gallastegi T, Soto B, Romero JM, Galán M, Escudero JR, Camacho M. MCAM/CD146

401 Which is Differentially Expressed in Vascular Diseases, is a Potential Biomarker in

402 Abdominal Aortic Aneurysm. European Journal of Vascular and Endovascular Surgery.

$403 \quad 2019 ; 58(6): \mathrm{e} 454$.

404 18. Wang Z, Yan X. CD146, a multi-functional molecule beyond adhesion. Cancer letters.

$405 \quad 2013 ; 330(2): 150-62$.

406 19. Milo R, Shen-Orr S, Itzkovitz S, Kashtan N, Chklovskii D, Alon U. Network motifs:

407 simple building blocks of complex networks. Science. 2002;298(5594):824-7.

408 20. Zhao Z, Li C, Zhang X, Chiclana F, Viedma EH. An incremental method to detect

409 communities in dynamic evolving social networks. Knowledge-Based Systems.

410 $2019 ; 163: 404-15$. 
411 21. Liu Y-Y, Slotine J-J, Barabási A-L. Controllability of complex networks. nature.

$412 \quad 2011 ; 473(7346): 167-73$.

413 22. Goh K-I, Cusick ME, Valle D, Childs B, Vidal M, Barabási A-L. The human disease

414 network. Proceedings of the National Academy of Sciences. 2007;104(21):8685-90.

415 23. Akat KM, Morozov P, Brown M, Gogakos T, Da Rosa JC, Mihailovic A, et al.

416 Comparative RNA-sequencing analysis of myocardial and circulating small RNAs in human

417 heart failure and their utility as biomarkers. Proceedings of the National Academy of

418 Sciences. 2014;111(30):11151-6.

419 24. Eicher JD, Wakabayashi Y, Vitseva O, Esa N, Yang Y, Zhu J, et al. Characterization of 420 the platelet transcriptome by RNA sequencing in patients with acute myocardial infarction.

$421 \quad$ Platelets. 2016;27(3):230-9.

422 25. Xiao M, Zheng WX, Jiang G, Cao J. Stability and bifurcation analysis of arbitrarily high423 dimensional genetic regulatory networks with hub structure and bidirectional coupling. IEEE 424 Transactions on Circuits and Systems I: Regular Papers. 2016;63(8):1243-54.

425 26. De Domenico M, Nicosia V, Arenas A, Latora V. Structural reducibility of multilayer 426 networks. Nature communications. 2015;6(1):1-9.

427 27. Cheng M, An S, Li J. Identifying key genes associated with acute myocardial infarction.

428 Medicine. 2017;96(42).

429 28. Ge WH, Lin Y, Li S, Zong X, Ge ZC. Identification of biomarkers for early diagnosis of 430 acute myocardial infarction. Journal of Cellular Biochemistry. 2018;119(1):650-8. 
432 journal of medical research. 2019;24(1):22.

433 30. Guo Y, Wu C, Guo M, Liu X, Keinan A. Gene-Based Nonparametric Testing of

434 Interactions Using Distance Correlation Coefficient in Case-Control Association Studies.

435 Genes. 2018;9(12):608.

436 31. Wang Y, Chi X, Meng D, editors. The Application of Network Structure Aanalysis in the

437 Study of Disease Mechanisms. 2019 IEEE International Conference on Bioinformatics and

438 Biomedicine (BIBM); 2019: IEEE.

439 32. Opsahl T. Triadic closure in two-mode networks: Redefining the global and local

440 clustering coefficients. Social Networks. 2013;35(2):159-67.

441 33. Wang Y, Kou Y, Meng D. Network Structure Analysis Identifying Key Genes of Autism

442 and Its Mechanism. Computational and mathematical methods in medicine. 2020;2020.

443 34. Galton F. Typical laws of heredity. III. Nature. 1877;15(389):512-4.

444 35. Jeong H, Tombor B, Albert R, Oltvai ZN, Barabási A-L. The large-scale organization of 445 metabolic networks. Nature. 2000;407(6804):651-4.

446 36. Jeong H, Mason SP, Barabási A-L, Oltvai ZN. Lethality and centrality in protein

447 networks. Nature. 2001;411(6833):41-2.

448 37. Barrett P, Topol EJ. TCT-639 NR4A3 as a gene expression marker of acute

449 atherosclerotic plaque rupture in STEMI. Journal of the American College of Cardiology.

$450 \quad$ 2013;62(18 Supplement 1):B194. 

and fibrosis defined by global mRNA profiling of the human left ventricular myocardium.

454 Journal of molecular and cellular cardiology. 2007;42(4):870-83.

39. Haubner BJ, Adamowicz-Brice M, Khadayate S, Tiefenthaler V, Metzler B, Aitman T, et NY). 2012;4(12):966.

40. Jiang Y, Feng Y-P, Tang L-X, Yan Y-L, Bai J-W. The protective role of NR4A3 in acute myocardial infarction by suppressing inflammatory responses via JAK2-STAT3/NF- $\mathrm{B}$ pathway. Biochemical and biophysical research communications. 2019;517(4):697-702. pleiotropic physiological roles. Nuclear receptor signaling. 2006;4(1):nrs. 04002. by IL - 6 and IL - 17. Glia. 2012;60(5):771-81.

466 CXCR4 and CCL20 pathways regulates tumor growth. PloS one. 2009;4(4):e5125.

467 44. Hosokawa Y, Shindo S, Hosokawa I, Ozaki K, Matsuo T. IL-6 trans-signaling enhances

468 CCL20 production from IL-1 $\beta$-stimulated human periodontal ligament cells. Inflammation. 2014;37(2):381-6. 

and CCL20 on cardiac fibrosis in patients with myocardial infarction and isoproterenoltreated rats. Journal of clinical medicine. 2019;8(5):659. circulating levels of chemokines CXCL10, CCL20 and CCL22 in patients with ischemic heart disease. Cytokine. 2016;83:147-57. early proinflammatory phase in fracture healing reveals strong immunomodulatory effects of CD146 - positive mesenchymal stromal cells. Journal of tissue engineering and regenerative medicine. 2019;13(8):1466-81. MMP-9 activity at the blood-brain barrier promotes chemokine-induced leukocyte migration.

482 Cell reports. 2015;10(7):1040-54.

49. McMorrow JP, Murphy EP. Inflammation: a role for NR4A orphan nuclear receptors?

484 Biochemical Society Transactions. 2011;39(2):688-93.

50. Prince LR, Prosseda SD, Higgins K, Carlring J, Prestwich EC, Ogryzko NV, et al. NR4A 486 orphan nuclear receptor family members, NR4A2 and NR4A3, regulate neutrophil number 487 and survival. Blood, The Journal of the American Society of Hematology. 2017;130(8):1014488 25.

489 51. Liu H, Liu P, Shi X, Yin D, Zhao J. NR4A2 protects cardiomyocytes against myocardial 490 infarction injury by promoting autophagy. Cell death discovery. 2018;4(1):1-11. 
491 52. Croker BA, Mielke LA, Wormald S, Metcalf D, Kiu H, Alexander WS, et al. Socs3

492 maintains the specificity of biological responses to cytokine signals during granulocyte and

493 macrophage differentiation. Experimental hematology. 2008;36(7):786-98.

494

495 Figure Legends

496 Figure 1. Flow chart for study design. DEGs, differential expression genes; DCGs, differential

497 connectivity genes.

498 Figure 2. Gene expression profiles of DEGs. 126 genes show significant differential

499 expressions between the AMI and the control groups in the discovery cohort (A) and validation

500 cohort (B), thus define as DEGs. DEGs, differential expression genes.

501 Figure 3. Assessment of DEGs' networks. Networks in the control and AMI groups are

502 independent and separable according to the average clustering coefficients of DEGs (A).

503 Number of clusters within DEGs' networks progressively decline when thresholds increase

504 from 0.1 to 0.9 (B). The AMI group has a lower decline slope. The gene networks of DEGs in

505 the AMI group has more complex connection compare to that in the control group (C).

506 Networks are present under threshold 0.5 and 0.7. Darker line represents connections under

507 threshold 0.7; lighter line represents connections under threshold 0.5. DEGs, differential

508 expression genes. 
509 Figure 4. Identification of DCGs. Genes that clustering coefficient increased over 0.1 in the

510 AMI group, in discovery cohort and validation cohort, are revealed as DCGs (A). Gene

511 expression profile of DCGs shows stable increase in AMI group in two cohorts (B). The

512 connection among DCGs in the AMI group are denser $(\mathbf{C})$ and the average degrees of DCGs

513 in AMI group are higher (D) compare to the control group in two cohorts. Networks are

514 presented under threshold 0.5 and 0.7 . Darker line represents connections under threshold 0.7;

515 lighter line represents connections under threshold 0.5. Degree are presented as mean $\pm \mathrm{SEM}$.

516 DCGs, differential connectivity genes.

517 Figure 5. Visualization of DCGs in DEGs' networks. The networks of DEGs in discovery

518 cohort (A) and in the validation cohort (B) indicate that the DCGs participate in distinctive

519 ways in the control group and in the AMI group. DCGs switch to central functional position of

520 networks and participate in more intricate connections under AMI situation. Yellow nodes

521 indicate the DCGs. Red gene names indicate the hub genes. DEGs, differential expression

522 genes; DCGs, differential connectivity genes.

523 Figure 6. Analysis of gene connection and expression of DCGs in discovery cohort. The

524 analysis of clustering coefficient and gene expression revealed CCL20 and NR4A3 as hub genes

525 (A). The CCL20 is a chemoattractant while $N R 4 A 3$ is a nuclear factor receptor (B). Subgraphs

526 of CCL20 and NR4A3 substantiate their important roles in AMI development (C). Networks

527 are presented under threshold 0.5 and 0.7. Darker line represents connections under threshold

5280.7 ; lighter line represents connections under threshold 0.5. DCGs, differential connectivity genes; CC, clustering coefficient; GeExp, gene expression. 
530 Figure 7. CCL20 cluster and NR4A3 cluster formation in early-stage AMI. CCL2O and NR4A3

531 stay in the peripheral position of DCGs' network under normal state (A). However, they shift

532 to the primary position of DCGs' network dominating two functional clusters under AMI

533 stimulation (B). DCGs, differential connectivity genes. 
Figures

\section{Figure 1.}

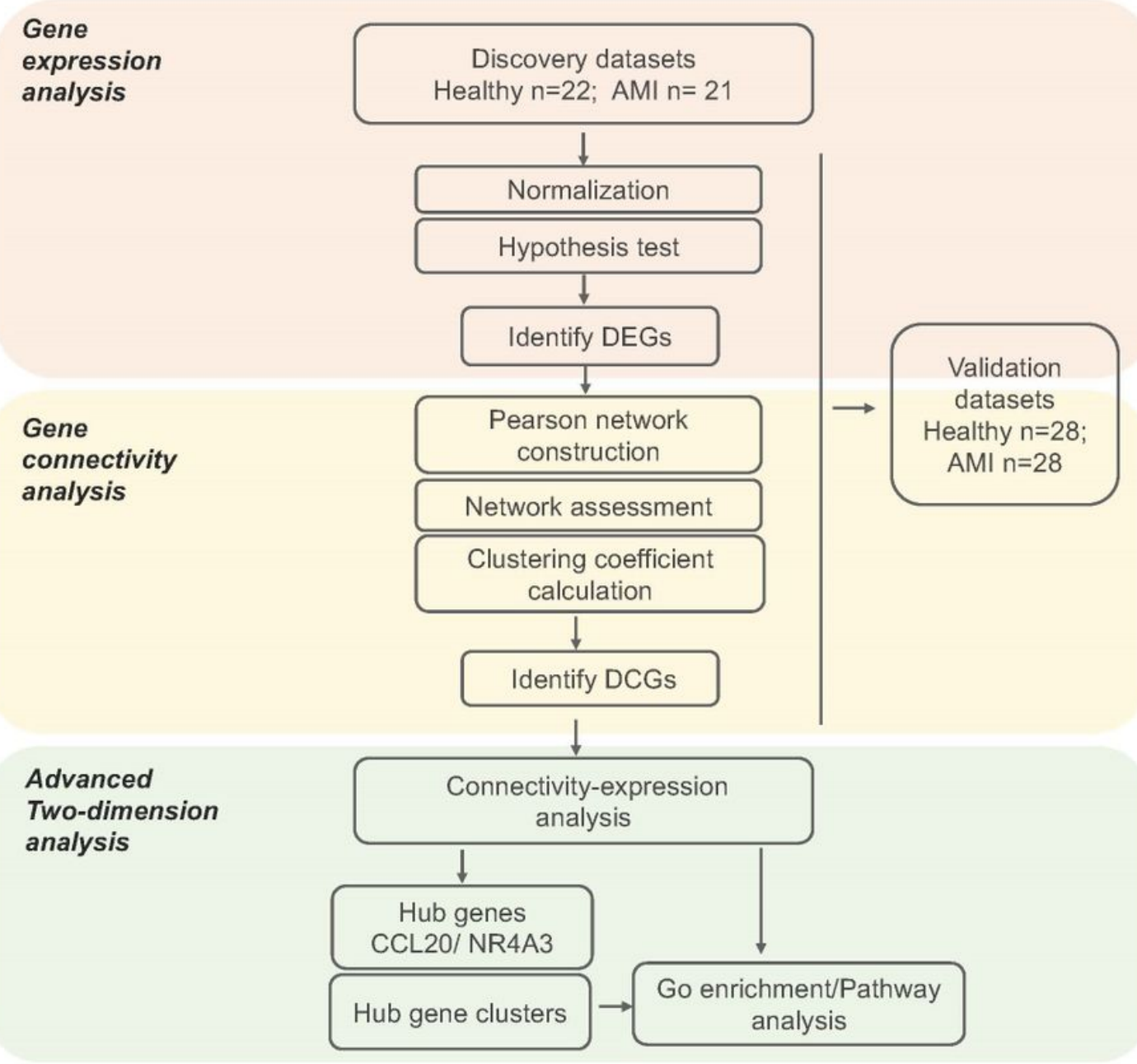

\section{Figure 1}

Flow chart for study design. DEGs, differential expression genes; DCGs, differential connectivity genes. 


\section{Figure 2.}

A

Discovery cohort
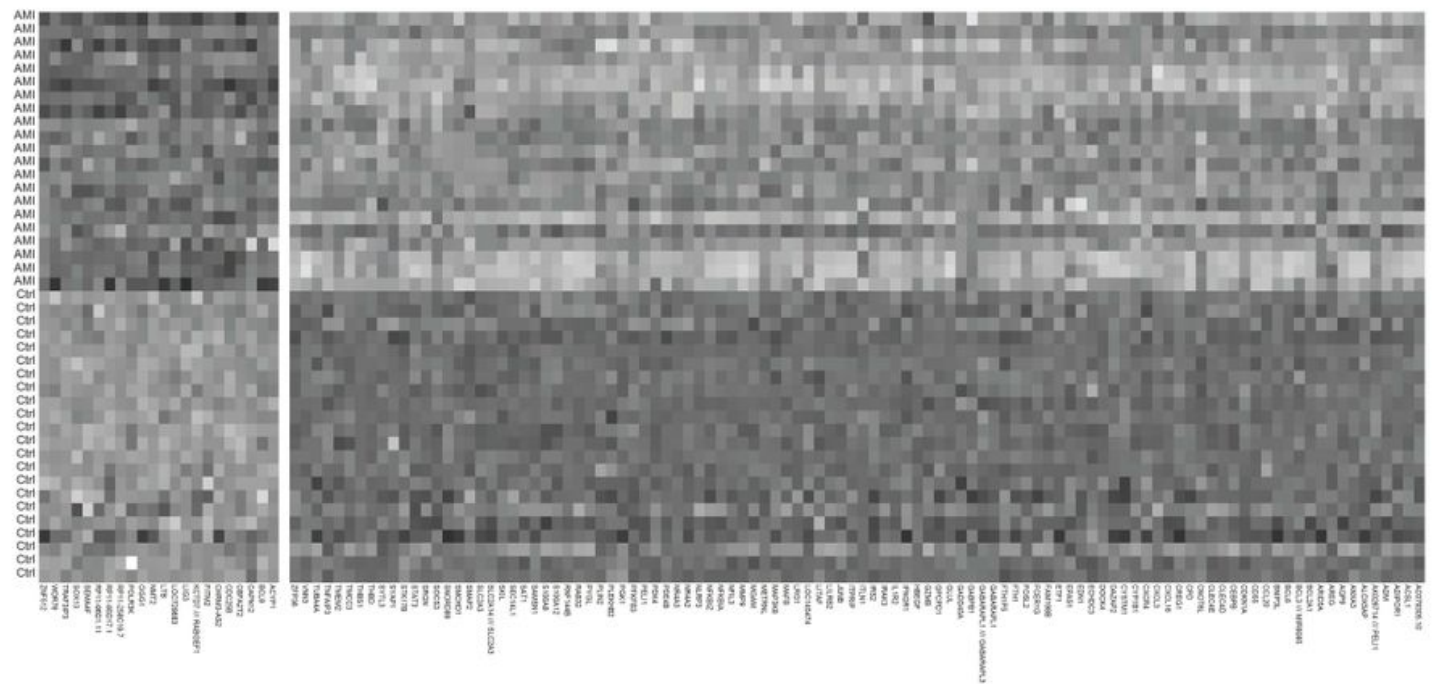

B

Validation cohort
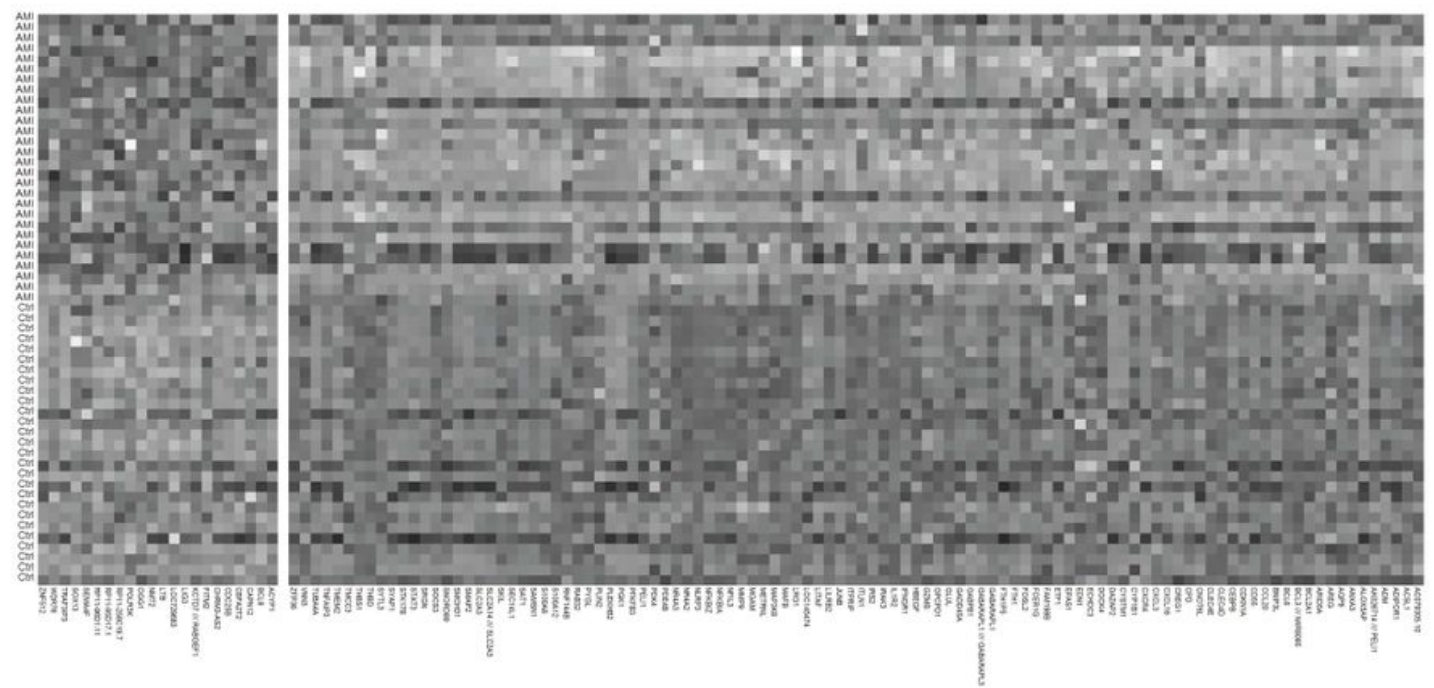

\section{Figure 2}

Gene expression profiles of DEGs. 126 genes show significant differential expressions between the AMI and the control groups in the discovery cohort (A) and validation cohort (B), thus define as DEGs. DEGs, differential expression genes. 
Figure 3.
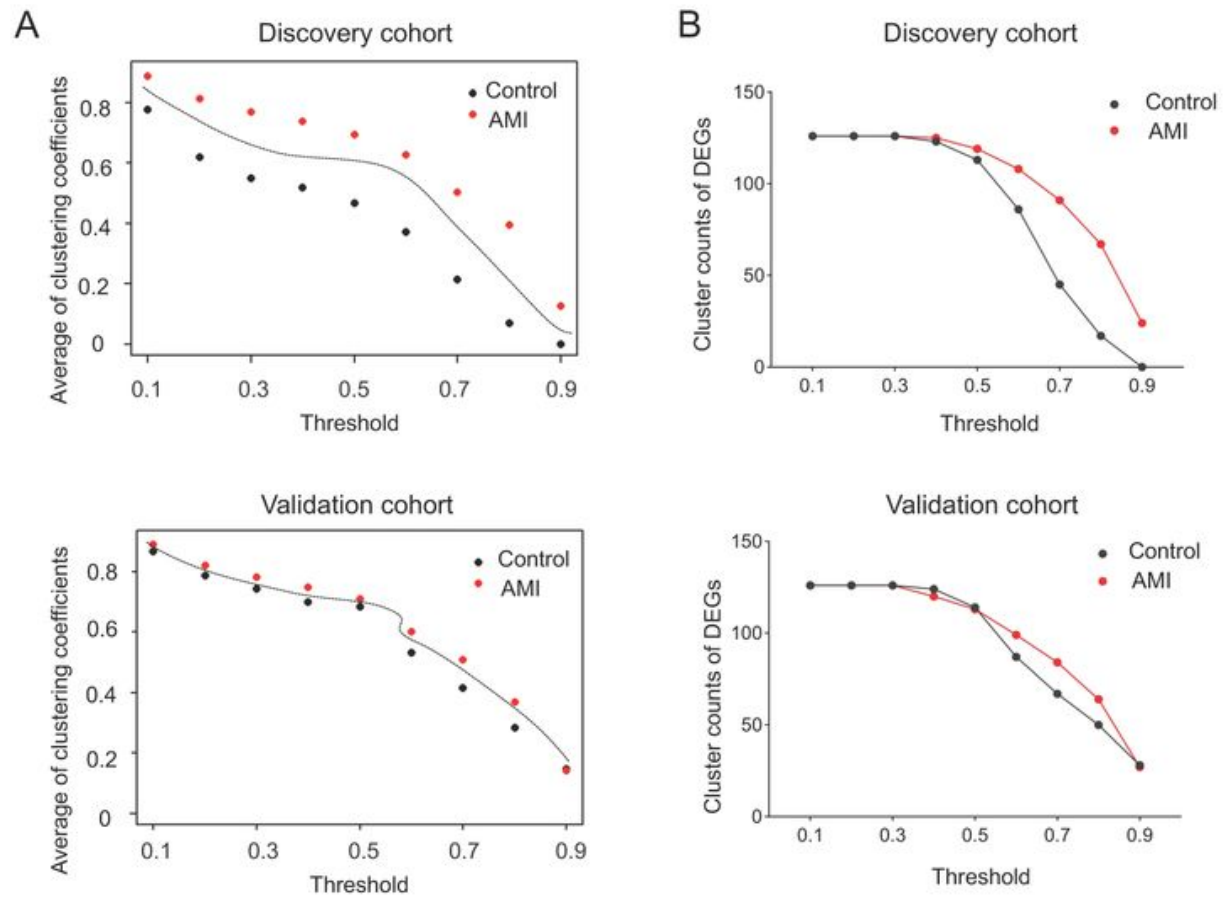

C

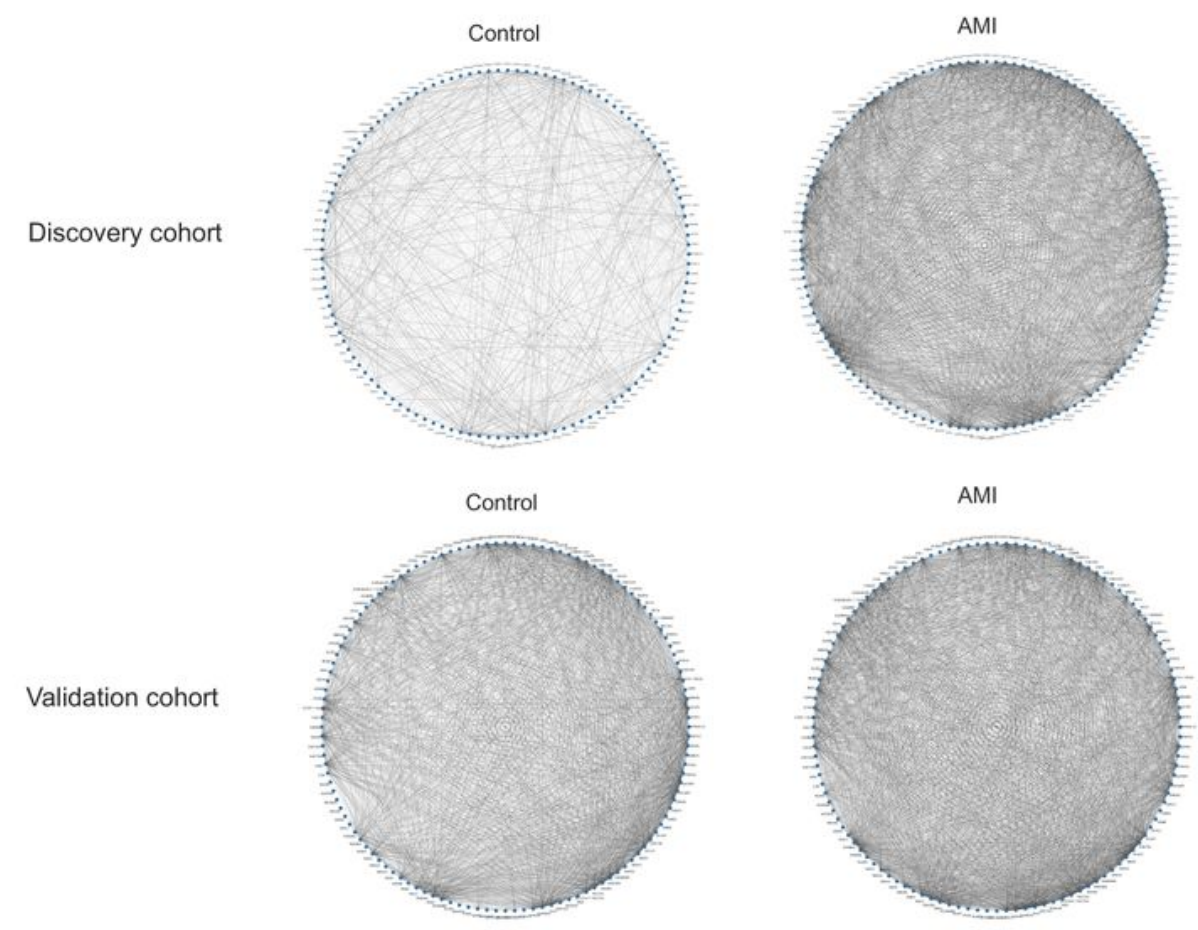

\section{Figure 3}

Assessment of DEGs' networks. Networks in the control and AMI groups are independent and separable according to the average clustering coefficients of DEGs (A). Number of clusters within DEGs' networks progressively decline when thresholds increase from 0.1 to 0.9 (B). The AMI group has a lower decline slope. The gene networks of DEGs in the AMI group has more complex connection compare to that in the control group (C). Networks are present under threshold 0.5 and 0.7. Darker line represents connections 
under threshold 0.7; lighter line represents connections under threshold 0.5. DEGs, differential expression genes.

Figure 4.

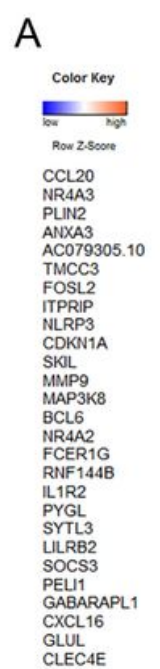

C
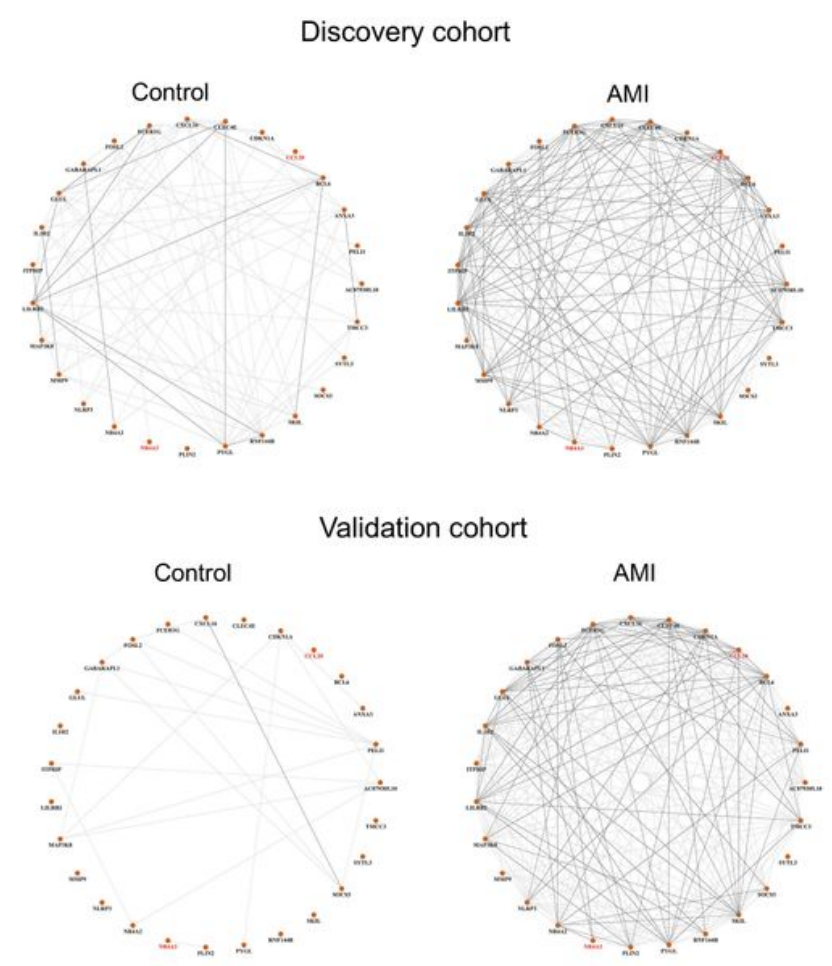

B

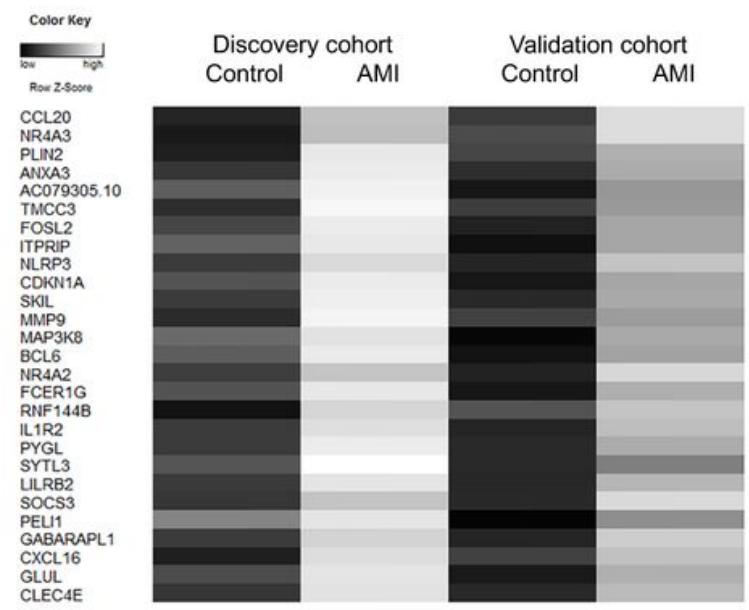

D
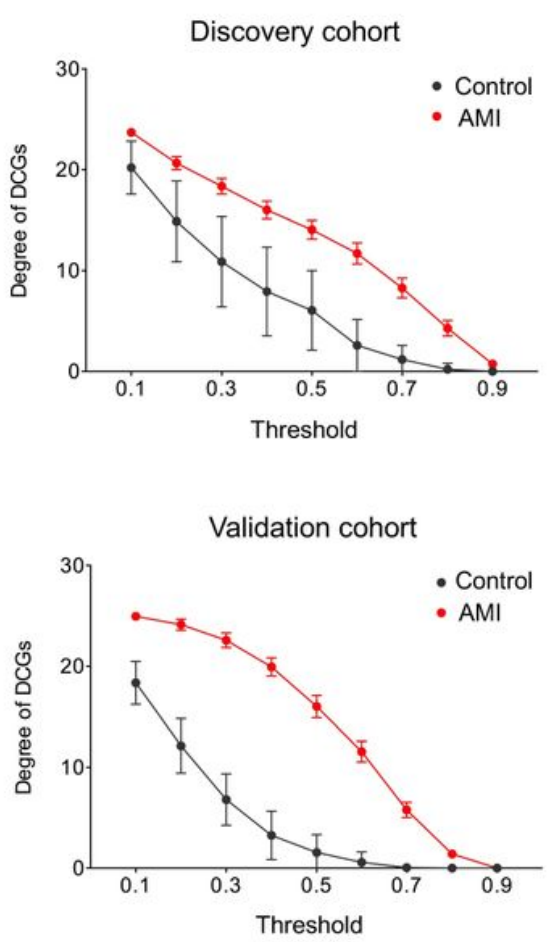

\section{Figure 4}

Identification of DCGs. Genes that clustering coefficient increased over 0.1 in the AMI group, in discovery cohort and validation cohort, are revealed as DCGs (A). Gene expression profile of DCGs shows stable increase in AMI group in two cohorts (B). The connection among DCGs in the AMI group are denser (C) and the average degrees of DCGs in AMI group are higher (D) compare to the control group in two 
cohorts. Networks are presented under threshold 0.5 and 0.7. Darker line represents connections under threshold 0.7; lighter line represents connections under threshold 0.5. Degree are presented as mean \pm SEM. DCGs, differential connectivity genes.

Figure 5.
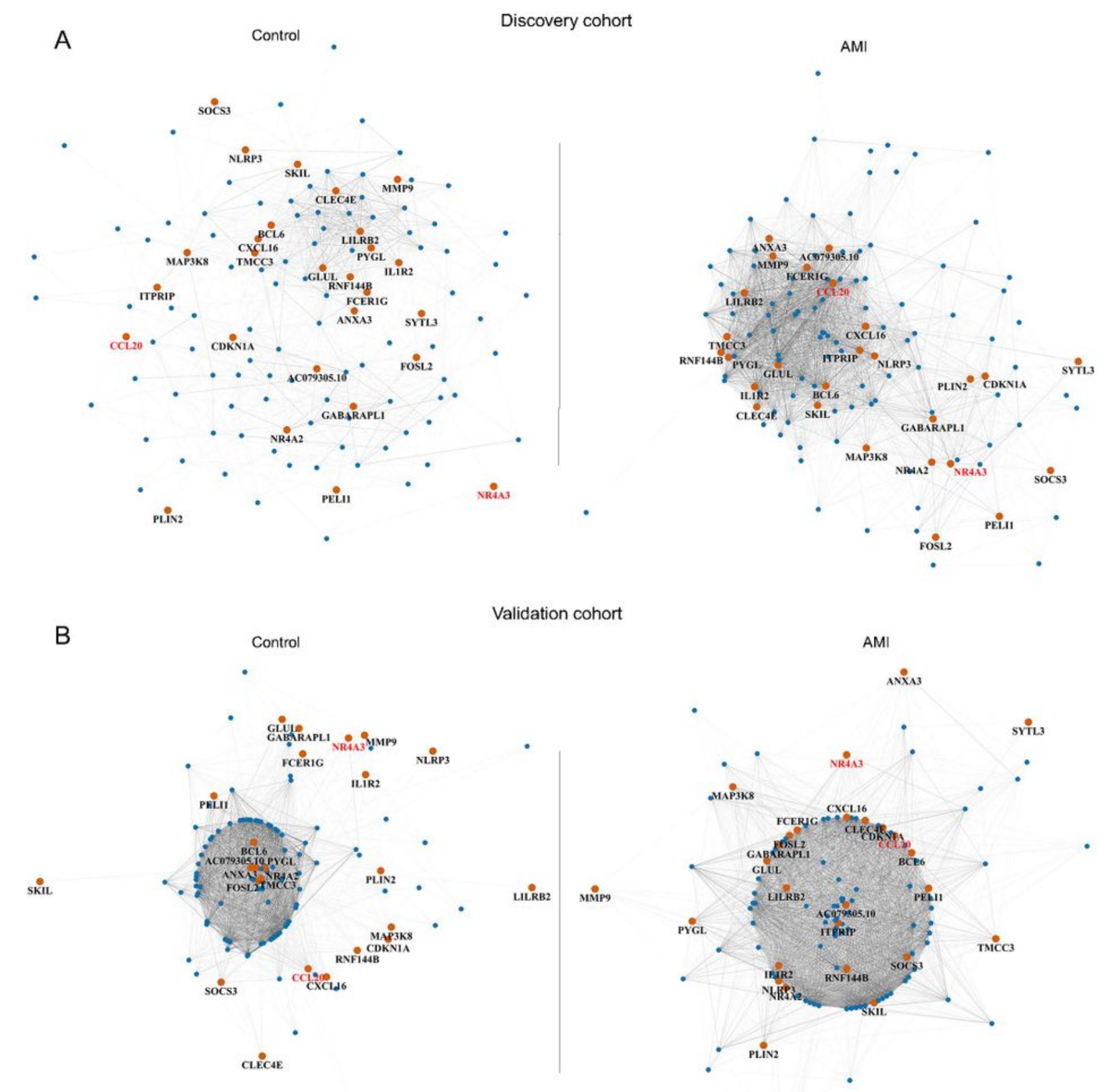

Validation cohort

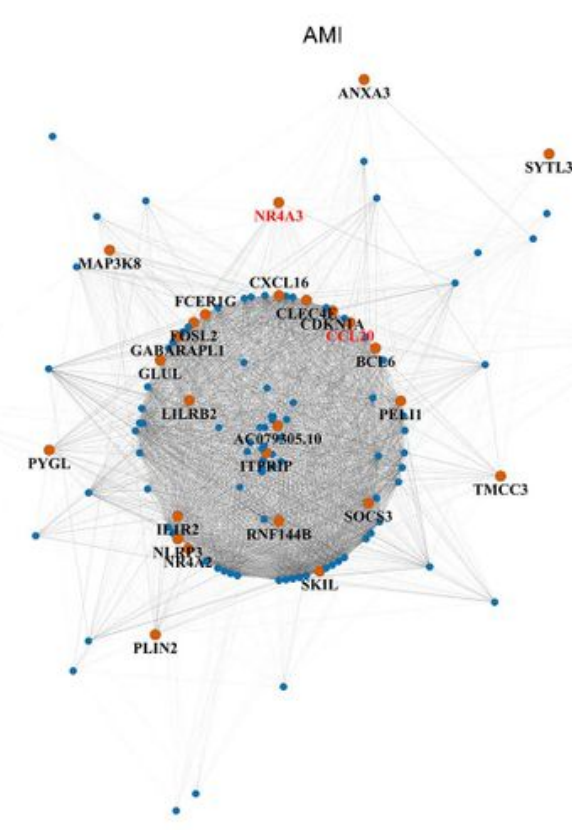

\section{Figure 5}

Visualization of DCGs in DEGs' networks. The networks of DEGs in discovery cohort (A) and in the validation cohort (B) indicate that the DCGs participate in distinctive ways in the control group and in the 
AMI group. DCGs switch to central functional position of networks and participate in more intricate connections under AMI situation. Yellow nodes indicate the DCGs. Red gene names indicate the hub genes. DEGs, differential expression genes; DCGs, differential connectivity genes.

Figure 6.

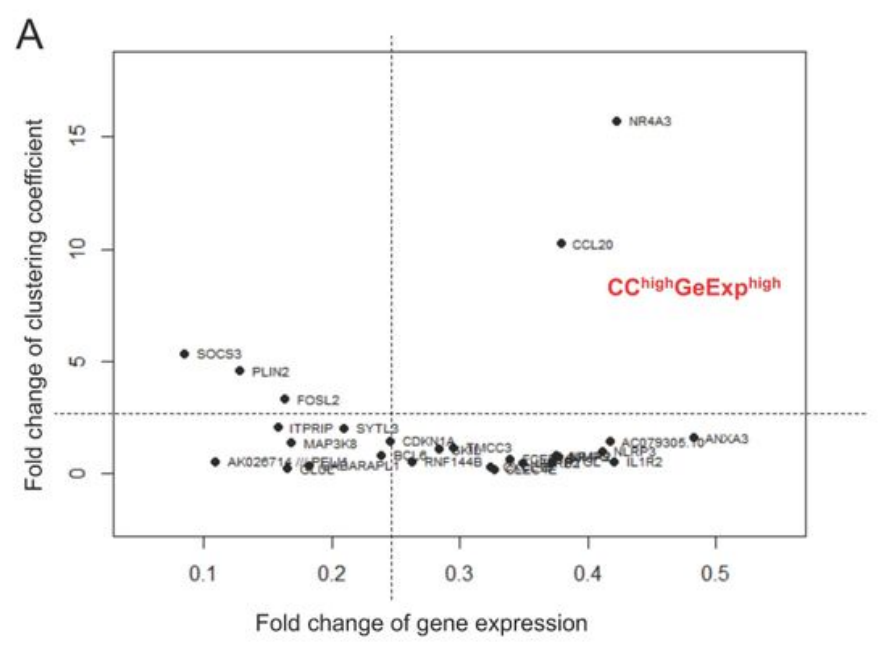

B

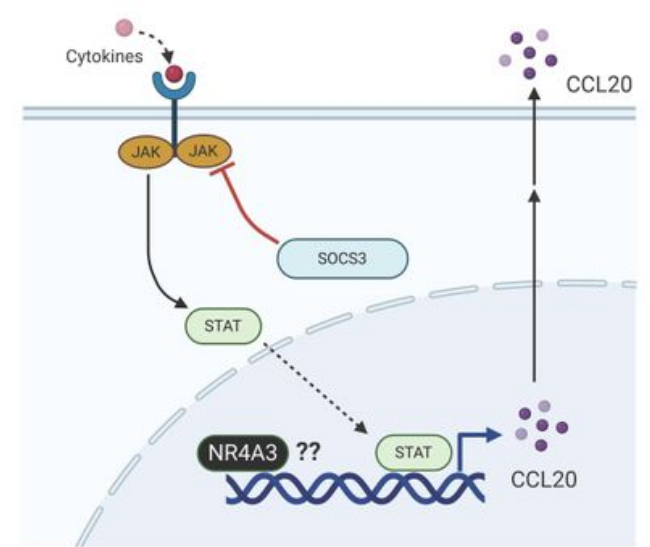

C

CCL20

NR4A3
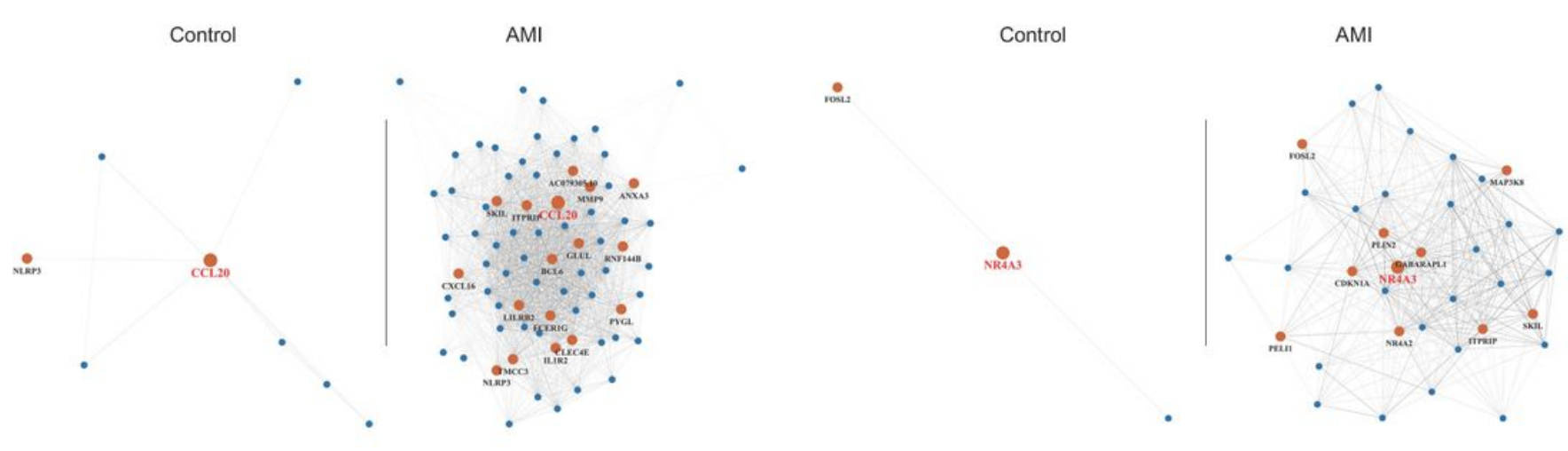

\section{Figure 6}

Analysis of gene connection and expression of DCGs in discovery cohort. The analysis of clustering coefficient and gene expression revealed CCL20 and NR4A3 as hub genes (A). The CCL20 is a chemoattractant while NR4A3 is a nuclear factor receptor (B). Subgraphs of CCL20 and NR4A3 substantiate their important roles in AMI development (C). Networks are presented under threshold 0.5 and 0.7. Darker line represents connections under threshold 0.7; lighter line represents connections under threshold 0.5. DCGs, differential connectivity genes; CC, clustering coefficient; GeExp, gene expression. 
Figure 7.

A Control

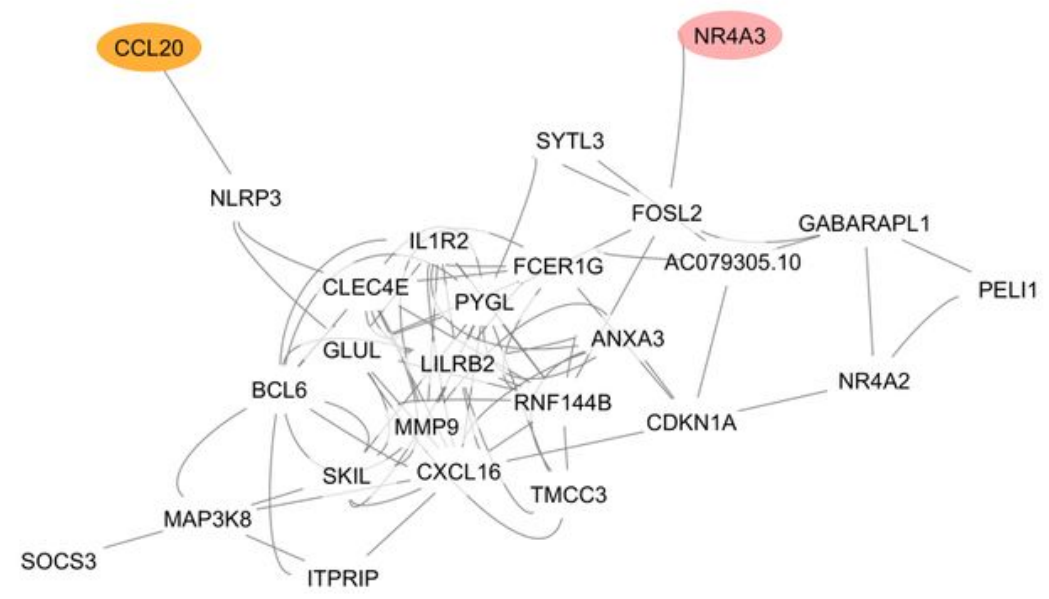

B AMI

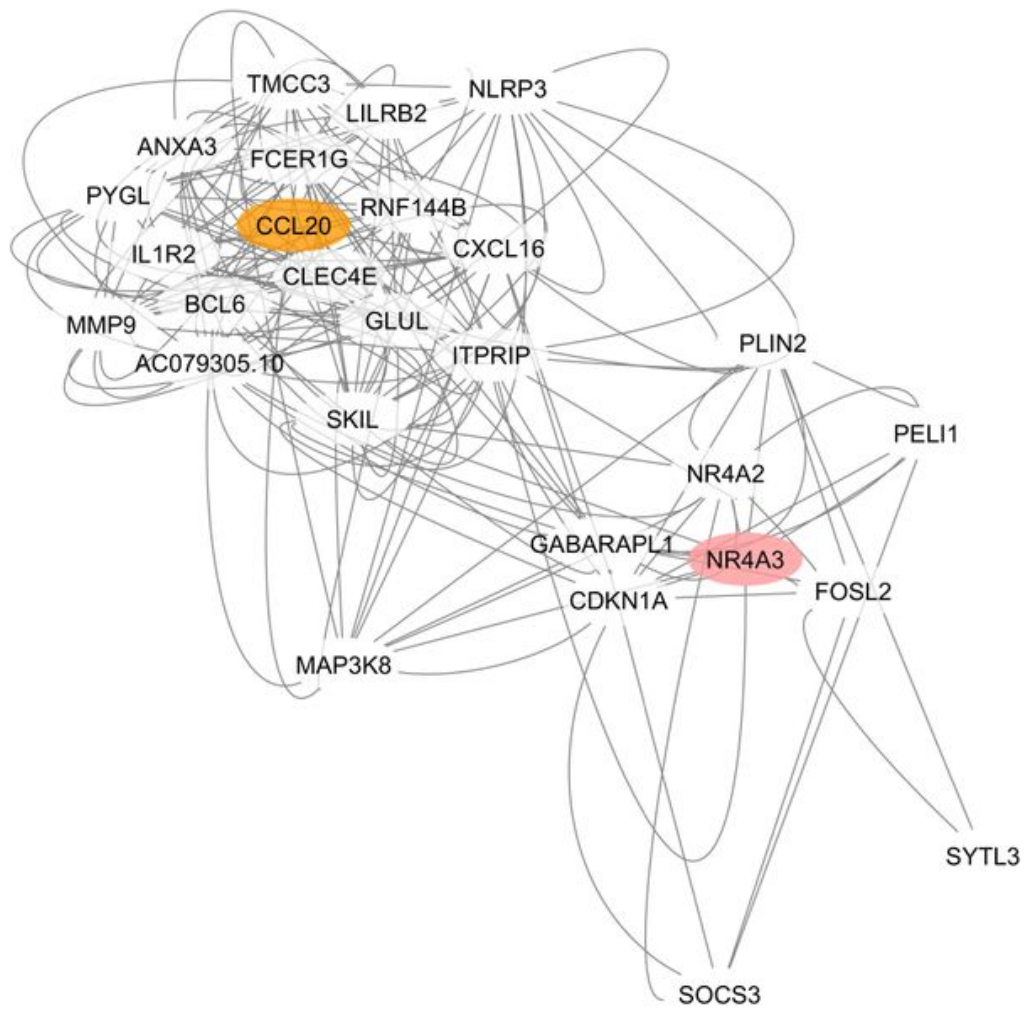

\section{Figure 7}

CCL20 cluster and NR4A3 cluster formation in early-stage AMI. CCL20 and NR4A3 stay in the peripheral position of DCGs' network under normal state (A). However, they shift to the primary position of DCGs' network dominating two functional clusters under AMI stimulation (B). DCGs, differential connectivity genes. 


\section{Supplementary Files}

This is a list of supplementary files associated with this preprint. Click to download.

- Tables.docx

- supplementaryfigures.pdf

- supplementarytables.pdf 